\title{
Genes, history and detail
}

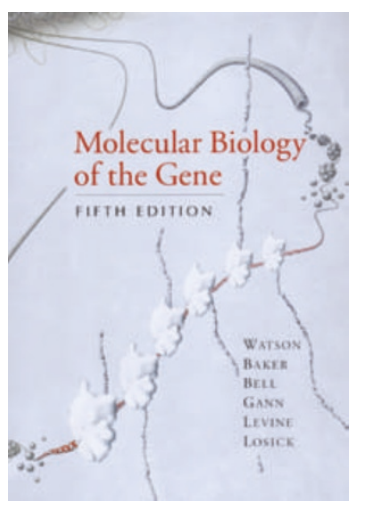

\section{Molecular Biology of the Gene, Fifth Edition}

by James D. Watson, Tania A. Baker, Stephen P. Bell, Alexander Gann, Michael Levine and Richard Losick

Cold Spring Harbor Laboratory

Press/Benjamin Cummings • 2004

$\$ 123 / £ 38.99$

\section{Peter F. R. Little}

To teach any biological science today is to steer a delicate course between science as history and science as research. The five authors of Molecular Biology of the Gene span the iconic past to the toiling present of a branch of science that perhaps, more than any other, blurs this distinction. This can create a barrier to understanding the past and building the future, a difficulty that is compounded by increasing evidence that even basic molecular definitions are now somewhat obsolete. For example, a decade ago genes were defined as transcriptional units that code for protein or functional RNA, but this definition does not adequately account for the extraordinary percentage of the vertebrate genome that is transcribed into RNA that may not be directly functional. In short, contemporary molecular biology is a subject full of hazards for the writers of a text book, with basic terminology becoming increasingly ambiguous and new technology making historical skills obsolete.

As if the task isn't already difficult enough, there is another even more pressing obstacle: the dominant role of molecular detail in describing gene control. The thinking that molecular mechanisms could be universal was perhaps at its zenith with the discovery of the Lac operon. At that moment, it seemed clear that if you could put together enough Lac operons, then you could control the genes of an elephant, for example. The next 30 years revealed that this was a seductive, but erroneous view; evolution does not work in this way and for the gene, the devil is indeed in the detail of multiple protein-protein, protein-DNA and DNA-DNA interactions. How can a textbook operate in this field? How, for example, can it range from a fundamental principle, such as DNA being the genetic material, to the staggering detail behind the control of segmentation genes? Principles, at this cutting edge, are such high-level generalizations as to be almost useless, and detail is often overwhelming or arbitrarily limited.

Against this backdrop, Molecular Biology of the Gene tackles the sub- ject well. This is achieved through a very careful structure, new to edition five, that separates the book into five distinct parts: chemistry and genetics, maintenance of the genome, expression of the genome, regulation, and finally methods. There are two reasons why this is critical to the success of the book: chemistry and genetics is the place for history and methods is the place to understand the technological drivers of molecular biology and the model organisms we use. At a stroke, this frees the author from being held hostage by some of the detail because, as they state in the introduction to the first edition: "Given the choice between deleting an important principal or giving experimental detail, I am inclined to state the principle".

The coverage of the key topics within each of these parts is comprehensive and beautifully illustrated with numerous figures that are also available in the accompanying $\mathrm{CD}$, a very useful feature that will provide key resources for both teachers and students. Particularly amusing are the pictures of key individuals, drawn from Cold Spring Harbor's wonderful archives, including a bevy of very youthful Nobel Laureates and my favourite image from 1961 of Monod and Szilard, perfectly illuminated in front of a glowing blackboard that shows the chalk marks of the then paradigm of genetic control. The selection of topics is conventional — how could it be otherwise? - but the coverage of some of the undoubted triumphs of developmental biology is particularly good.

There is no doubt that this is an excellent text book; mature, beautifully discussed and illustrated. But the usual genes appear and vanish leaving a slight feeling of 'yes, that was fine, but how does it all fit together?'. This is perhaps the most unsatisfactory part of molecular biology as a discipline of the $20^{\text {th }}$ century, and this textbook is poised on the edge of yet another great change as the thinking and techniques of systems biology become increasingly significant. The approach to biology in the $21^{\text {st }}$ century may no longer be a reductionist one, and it is going to be fascinating to see how well we succeed at deciphering the staggering detail that three billion years of evolution has created within the cells of our bodies. Unfortunately, I do not believe this book brings to a sharp focus what the genome projects have really achieved so far; not the 'blueprint of human beings' or the hyperbole of genetic prediction, but rather the creation of the tools and thinking that will power the new approach of systems biology and with which we will attempt to confront the real complexity of living systems.

Textbooks, as the trite aphorism goes, are out of date as soon as they are published; of course a textbook cannot be a review of the edges of the subject. If it is really to educate, however, it must leave the student at those edges, both informed and inspired. By this measure, the fifth edition of Molecular Biology of the Gene succeeds with great clarity and verve in most areas of molecular biology, but fails where the genomic revolution starts. I believe the fifth edition is one of the very best textbooks in a crowded field; it is perfectly poised to educate and to inform, but we may have to await the sixth edition to be truly inspired. 